\title{
Near-field radiative heat transfer between a sphere and a substrate
}

\author{
Arvind Narayanaswamy \\ Department of Mechanical Engineering, Columbia University, New York, New York 10027, USA
}

\author{
Sheng Shen and Gang Chen \\ Department of Mechanical Engineering, Massachusetts Institute of Technology, Cambridge, Massachusetts 02139, USA
}

(Received 20 June 2008; published 4 September 2008)

\begin{abstract}
Near-field force and energy exchange between two objects due to quantum electrodynamic fluctuations give rise to interesting phenomena such as Casimir and van der Waals forces and thermal radiative transfer exceeding Planck's theory of blackbody radiation. Although significant progress has been made in the past on the precise measurement of Casimir force related to zero-point energy, experimental demonstration of near-field enhancement of radiative heat transfer is difficult. In this work, we present a sensitive technique of measuring near-field radiative transfer between a microsphere and a substrate using a bimaterial atomic force microscope cantilever, resulting in "heat transfer-distance" curves. Measurements of radiative transfer between a sphere and a flat substrate show the presence of strong near-field effects resulting in enhancement of heat transfer over the predictions of the Planck blackbody radiation theory.
\end{abstract}

DOI: 10.1103/PhysRevB.78.115303

PACS number(s): 87.64.Dz, 44.40.+a, 03.50.De, 05.40.-a

Though Planck ${ }^{1}$ realized that the theory of blackbody radiation is applicable only to objects with characteristic dimensions larger than the wavelength of thermal radiation, a rigorous theory of near-field radiative transfer was established only later by Polder and Van Hove ${ }^{2}$ and others ${ }^{3-8}$ following the fluctuational electrodynamics formalism established by Rytov. ${ }^{9}$ The modification of thermal fluctuations of the electromagnetic field due proximity between two objects is the source of near-field thermal radiative transfer as well as thermal contributions to dispersion forces such as Casimir and van der Waals forces. Experimentally, there are a few reports $^{10-13}$ on near-field heat transfer experiments, but none has demonstrated exceeding Planck's blackbody radiation law. ${ }^{14}$ Domoto et al. ${ }^{10}$ reported increased near-field radiative heat transfer at cryogenic temperatures between two parallel metallic plates at gaps of $50 \mu \mathrm{m}$ to $1 \mathrm{~mm}$, but the total heat transfer is $\approx 1 / 29$ of the Planck theory prediction. Hargreaves ${ }^{11}$ extended the measurements, between two chromium surfaces, to gaps up to $1 \mu \mathrm{m}$ at room temperature, demonstrating an enhancement from $\approx 1.75 \mathrm{~W} \mathrm{~m}^{-2} \mathrm{~K}^{-1}$ in the far field to $\approx 2.95 \mathrm{~W} \mathrm{~m}^{-2} \mathrm{~K}^{-1}$ at $1 \mu \mathrm{m}$. However, the near-field radiation heat transfer between two chromium surfaces is still less than $50 \%$ of blackbody radiation. Xu et al. ${ }^{12}$ could not measure any signature of near-field enhancement because of the low sensitivity of their experimental technique. Recently, Kittel et al. ${ }^{13}$ investigated the near-field radiative transfer between a sharpened scanning tunneling microscope tip and a flat substrate and showed a saturation of heat transfer at gaps of $10 \mathrm{~nm}$ or less and a decrease at larger gaps. The saturation effects and enhancement of heat transfer have been attributed to spatial dispersion effects and the contribution of the infrared magnetic-dipole component. ${ }^{15,16}$ However, the complicated geometry of the tip makes it difficult to interpret the experimental data. More precise measurements are needed to confirm past extensive theoretical studies and predictions of near-field enhancement, especially that of exceeding Planck's theory of blackbody radiation. Such near-field experiment also provides insight into thermal contributions to the Casmir force, which has been elusive to experimental detection because of its much smaller magnitude compared to the contribution of the zero-point energy. ${ }^{17,18}$

We decided to measure radiative transfer between a microsphere and a flat substrate in order to overcome the difficulties encountered in the past experiments and use bimaterial atomic force microscopy (AFM) cantilevers as thermal sensors. Bimaterial cantilevers bend in response to changes in temperature distribution in the cantilever due to the difference in coefficient of thermal expansion of the two materials comprising the cantilever. They have been used as sensitive calorimeters $^{19,20}$ and IR detectors. ${ }^{21,22}$ Such cantilevers are reported to have a minimum measurable temperature of $10^{-4}-10^{-5} \mathrm{~K}$ and a minimum detectable power of $5 \times 10^{-10} \mathrm{~W}$ (when optimized, the minimum detectable levels are even lower). ${ }^{20}$ With a temperature difference of $50 \mathrm{~K}$ between the sphere and the substrate, the minimum detectable conductance is $\approx 10^{-11} \mathrm{~W} \mathrm{~K}^{-1}$. A similar configuration has also been used for high-precision measurements of $\mathrm{Ca}$ simir force. ${ }^{17,23}$ Using the rigorous theory for near-field radiative transfer between two spheres that we have developed elsewhere, ${ }^{8}$ it is possible to estimate the values of thermal conductance between a sphere and a substrate. ${ }^{24}$ This theory predicts that the conductance between two silica spheres with diameters of $50 \mu \mathrm{m}$ ranges from $\approx 10^{-9}$ to $\approx 10^{-8} \mathrm{~W} \mathrm{~K}^{-1}$ for gaps between $100 \mathrm{~nm}$ and $10 \mu \mathrm{m}$, well within the measurement limits of the bimaterial cantilever. The near-field enhancement is due to the tunneling of surface phonon polaritons present at interfaces between silica and vacuum due to thermal fluctuations of the electromagnetic field. Hence we have chosen silica spheres with diameter of $50 \mu \mathrm{m}$ for our experiment.

A schematic of the experimental apparatus is shown in Fig. 1(a) and a silica sphere is attached to the tip of a triangular $\mathrm{SiN} / \mathrm{Au}$ cantilever (from BudgetSensors) as shown in Fig. 1(b). To decrease the influence of dispersion and electrostatic forces, the cantilever is oriented perpendicular to the substrate $\left(90^{\circ} \pm 2^{\circ}\right)$. The substrate, which is rigidly attached to the motion control stage, is a glass microscope slide. The 

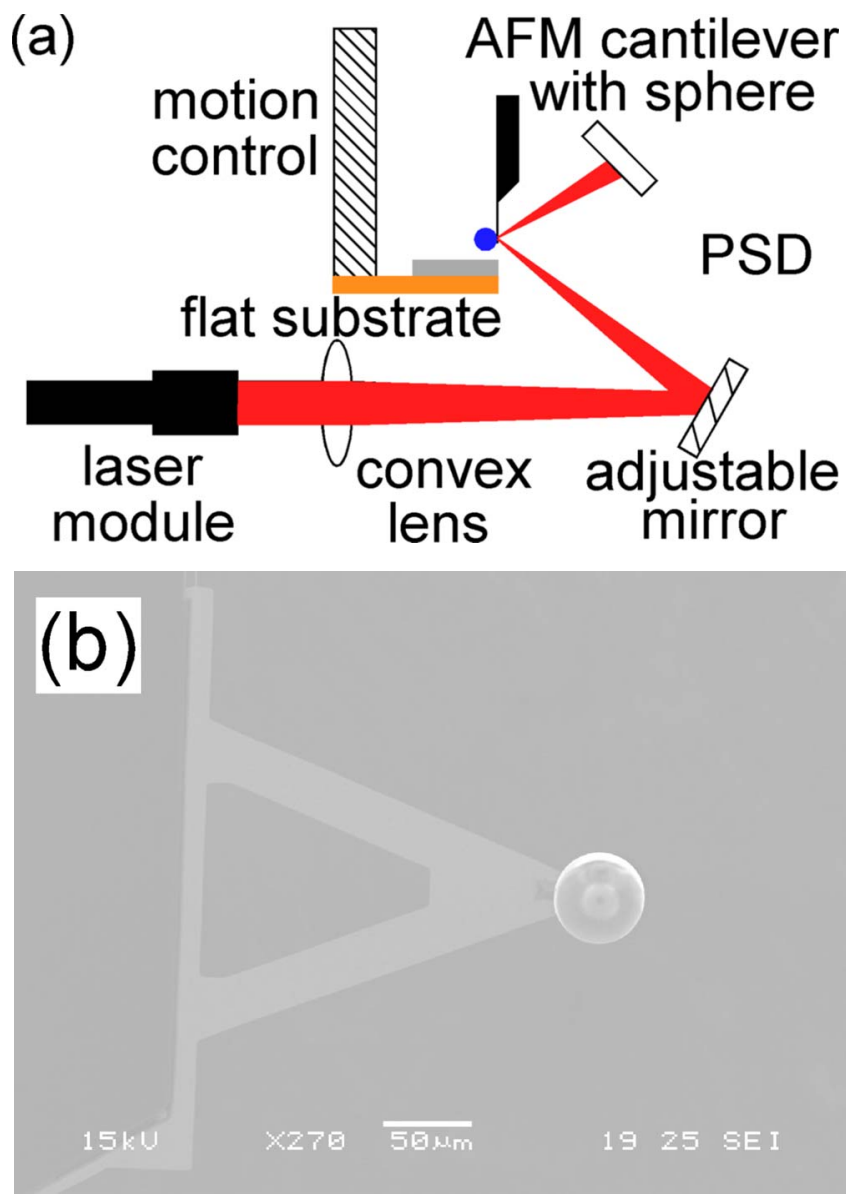

FIG. 1. (Color online) (a) Schematic of experimental apparatus. The beam from a laser diode module is focused at the tip of an AFM cantilever and the reflected portion is directed onto a PSD. The beam from the adjustable mirror onto the cantilever is the "incident beam," and the beam from the cantilever to the PSD is the "reflected beam." (b) Scanning electron microscopy (SEM) image of silica sphere attached to the tip of a triangular bimaterial cantilever.

apparatus is placed inside a vacuum chamber pumped down to $\approx 6.7 \times 10^{-3} \mathrm{~Pa}$ during the experiment. The laser beam is focused at the tip of the cantilever and the reflected beam forms a spot on a position sensitive detector (PSD). The position of the spot and the laser power incident on the PSD are obtained from the PSD difference and sum signals, respectively. The change in position of this spot is a measure of the deflection of the cantilever. A portion of the laser beam is absorbed by the cantilever and results in a temperature rise of the cantilever tip and sphere. The base of the cantilever, the substrate, and the rest of the apparatus are approximately at the same temperature. As the gap between the sphere and the substrate decreases, increased heat transfer between the sphere and the substrate results in a cooling of the cantilever. The resultant deflection is measured as a change in the PSD difference signal, resulting in a "heat transfer-distance" curve. When the pressure inside the vacuum chamber is less than $0.1 \mathrm{~Pa}$, the dominant form of heat transfer mechanism is radiative transfer. As the radiation view factor from the sphere to the surrounding is $\approx 1$, and the substrate is at the
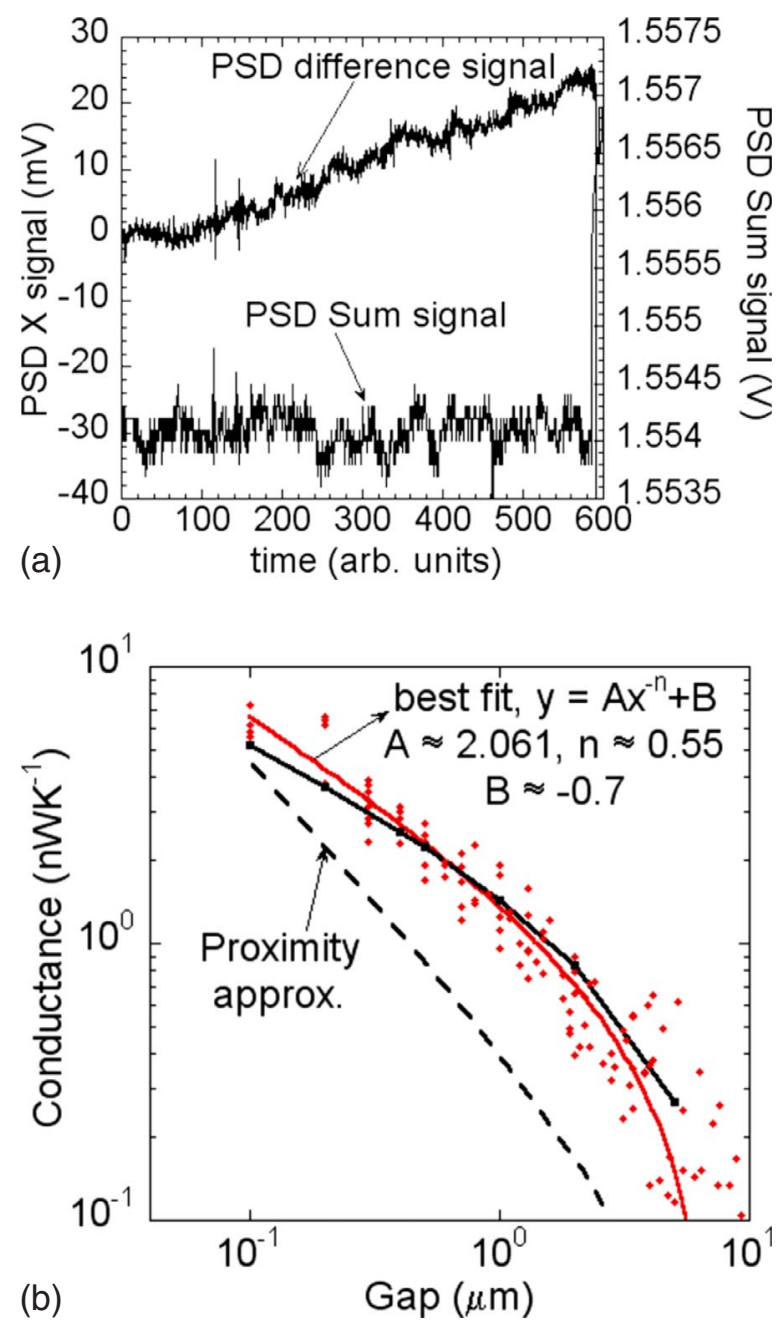

FIG. 2. (Color online) (a) Raw experimental data from one of the experiments. The $y$ axis to the left corresponds to the PSD difference signal and that on the right corresponds to the PSD sum signal. The substrate is brought closer to the sphere (smallest step size is $100 \mathrm{~nm}$ ) as the experiment proceeds. The contact is seen as a large change in the PSD signals. The sum signal is approximately a constant and this ensures that the deflection of the cantilever is not because of any spurious effect related to a change in the incident radiant power. (b) Experimental data (diamonds) from 13 heat transfer-distance measurements. The red/gray line through the data is a best fit curve of the form $y=A x^{-n}+B$. Also shown in the figure are the predictions of the proximity approximation (black dashed line) and the two-sphere problem (Refs. 8 and 26) predictions for the near-field transfer between a sphere and a flat plate (black line with black squares), obtained by multiplying the results of the twosphere problem by 2. (The factor of 2 is chosen because the conductance between a sphere and a flat substrate at a given gap is twice the conductance between two spheres of the same radii and gap.) The experimental configuration described in the text automatically measures the enhancement in radiative transfer due to nearfield and diffraction effects, which are not included in Planck's theory of blackbody radiation, relative to the value at $\approx 9 \mu \mathrm{m}$.

same temperature as the environment, there is no change in the far-field radiative heat transfer even when the cantilever approaches the substrate. Hence, what is measured in the heat transfer-distance curve is exclusively the near-field en- 
hancement above the far-field radiative transfer between two objects predicted by Planck's theory of radiative transfer.

To determine the conductance of radiative transfer between the sphere and the flat substrate, three quantities are necessary: (1) the heat absorbed by the cantilever (from the laser), (2) the heat transfer between the sphere and substrate, and (3) the temperature of the sphere. The absorptivity of the cantilever, determined by measuring the radiant power in the incident, transmitted, and reflected laser beams using the sum signal of the PSD, is approximately 0.13. The deflection of the cantilever is converted to heat transfer between the sphere and the substrate by determining the sensitivity of the cantilever to power absorbed at the tip. This sensitivity is determined by varying the radiant power of the incident laser beam. Knowing the amount of absorbed power at the tip, the temperature of the sphere is determined if the conductance of the cantilever, $G$, is known. This conductance is determined from the ratio of the sensitivity of the cantilever to uniform temperature rise of the cantilever to the sensitivity of the cantilever to power absorbed at the tip, as this ratio equals $2 G .^{20,25}$ For the cantilever used in this work, the sensitivity to heat transfer from the tip is measured to be $9.28 \times 10^{4} \mathrm{~V} \mathrm{~W}^{-1}$ and the sensitivity to uniform temperature change is $0.839 \mathrm{~V} \mathrm{~K}^{-1}$, resulting in a conductance of $4.52 \times 10^{-6} \mathrm{~W} \mathrm{~K}^{-1}$. With an incident power of $1.66 \mathrm{~mW}$ (as measured by a power meter), the absorbed power is $\approx 0.21 \mathrm{~mW}$ and the temperature rise of the tip, which is also the temperature rise of the sphere, is $46.5 \mathrm{~K}$.

Near-field effects become noticeable when the gap is approximately $10 \mu \mathrm{m}$ or less. ${ }^{8}$ The raw data from one of the experiments are shown in Fig. 2(a). Shown in the figure are two curves corresponding to the deflection signal ( $y$ axis to the left) and the sum signal ( $y$ axis to the right). As time increases along the $x$ axis, the gap between the sphere and the substrate decreases. Contact between the sphere and the substrate manifests itself as a large change in the PSD signals. Once the contact point is known, the position of the substrate can be converted to an equivalent gap. The sum signal curve is flat even as the gap decreases. This ensures that the signal that is measured is predominantly due to the near-field effect and not due to any spurious effects. ${ }^{26}$ The data from 13 heat transfer-distance curves are shown in Fig. 2(b). Each red/gray diamond marker in Fig. 2(b) corresponds to a data point in a heat transfer-distance curve. The scatter in the experimental data is $0.44 \mathrm{nW} / \mathrm{K}$ and is primarily because of the vibrations induced by the turbomolecular pump. In addition, the error bar in the $x$ axis due to the positional accuracy of the translation stage should be $100 \mathrm{~nm}$. To understand the experimental data, we point out that the heat transfer-distance curve measures only the near-field effect. Hence, the data in Fig. 2(b) correspond to the increase in near-field radiative transfer from the value at $\approx 9 \mu \mathrm{m}$.

We have used Mie theory ${ }^{27}$ to calculate the emissivity of a silica sphere with radius of $25 \mu \mathrm{m}$ to be 0.97 . Using this value of emissivity, the far-field conductance between the sphere and the substrate is approximately equal to the blackbody conductance between the sphere and the substrate. Hence the maximum measured conductance due to near-field enhancement, as seen in Fig. 2(b), is $6 \mathrm{nW} \mathrm{K}^{-1}$ above the prediction of Planck's theory of blackbody radiation value of $29 \mathrm{nW} \mathrm{K}^{-1} \cdot{ }^{28}$ From the analysis of near-field radiative transfer between two spheres, we see that the near-field conductance between the two spheres varies as $A x^{-n}$, where $n$ is an exponent less than 1 . If $n=1$, then the proximity approximation, which is widely used in determining forces between smoothly curved surfaces based on the results of forces between parallel surfaces, ${ }^{29,30}$ would be valid. Since the experimental measurements are relative to the gap from which measurements are started, the experimental data should be of the form $A x^{-n}+B$, where $B<0$. For the data shown in Fig. 2(b), the values of $n, A$, and $B$ are $0.55,2.061$, and -0.7 , in reasonable agreement with the numerical solution of the twosphere which yields values of $0.41,2.41$, and -0.978 for $n$, $A$, and $B$, respectively. ${ }^{26}$ It is also clear from Fig. $2(\mathrm{~b})$ that the experimental data are larger than the predictions of the proximity approximation, pointing to the lack of validity of the proximity approximation for near-field radiative heat transfer. We have shown that a proximity approximation-type theory is valid for those spheres where near-field effects dominate radiative transfer. ${ }^{8}$ This condition is satisfied only by silica spheres with diameter of less than $\approx 2 \mu \mathrm{m}$ and clearly not valid for microspheres with diameter of $\approx 50 \mu \mathrm{m}$.

In summary, we have introduced a sensitive technique based on bimaterial cantilevers to investigate near-field radiative transfer and report experimental data on radiative heat transfer between a silica sphere and a silica substrate. Our experimental technique is sensitive to near-field radiation alone. Our experimental data show the breakdown of the Planck blackbody radiation law in the near field and also show that proximity approximation cannot be applied to near-field radiation in the range of gaps involved in the experiment. Improvements in the current experimental setup ${ }^{26}$ can yield better measurements, enabling us to investigate near-field heat transfer between spheres of smaller diameter as well as between conducting spheres. The present experiment should also be of great interest for probing the temperature dependent behavior of the Casimir force.

This work was supported by DOE (Grant No. DE-FG0202ER45977) and ONR (Grant No. N00014-03-1-0835). The authors would also like to thank $\mathrm{Lu} \mathrm{Hu}$ for calculating the emissivity of silica spheres. 
${ }^{1}$ M. Planck, The Theory of Heat Radiation (Dover, New York, 1991).

${ }^{2}$ D. Polder and M. Van Hove, Phys. Rev. B 4, 3303 (1971).

${ }^{3}$ J. B. Pendry, J. Phys.: Condens. Matter 11, 6621 (1999).

${ }^{4}$ J. P. Mulet, K. Joulain, R. Carminati, and J. J. Greffet, Appl. Phys. Lett. 78, 2931 (2001).

${ }^{5}$ A. I. Volokitin and B. N. J. Persson, Phys. Rev. B 63, 205404 (2001).

${ }^{6}$ J.-P. Mulet, K. Joulain, R. Carminati, and J. J. Greffet, Microscale Thermophys. Eng. 6, 209 (2002).

${ }^{7}$ A. Narayanaswamy and G. Chen, Appl. Phys. Lett. 82, 3544 (2003).

${ }^{8}$ A. Narayanaswamy and G. Chen, Phys. Rev. B 77, 075125 (2008).

${ }^{9}$ S. M. Rytov, Theory of Electric Fluctuations and Thermal Radiation (Air Force Cambridge Research Center, Bedford, MA, 1959).

${ }^{10}$ G. A. Domoto, R. F. Boehm, and C. L. Tien, ASME Trans. J. Heat Transfer 92, 412 (1970).

${ }^{11}$ C. M. Hargreaves, Philips Res. Rep., Suppl. 5, 1 (1973).

${ }^{12}$ J. B. Xu, K. Lauger, R. Moller, K. Dransfeld, and I. H. Wilson, J. Appl. Phys. 76, 7209 (1994).

${ }^{13}$ A. Kittel, W. Muller-Hirsch, J. Parisi, S. Biehs, D. Reddig, and M. Holthaus, Phys. Rev. Lett. 95, 224301 (2005).

${ }^{14}$ Though the measured flux in these cases is less than the radiative conductance between two blackbody emitters at the same temperatures, they are greater than the far-field value predicted by taking the emissivity of the emitters into account.

${ }^{15}$ P. O. Chapuis, S. Volz, C. Henkel, K. Joulain, and J. J. Greffet, Phys. Rev. B 77, 035431 (2008).

${ }^{16}$ P. O. Chapuis, M. Laroche, S. Volz, and J. J. Greffet, Phys. Rev. B 77, 125402 (2008).
${ }^{17}$ S. K. Lamoreaux, Phys. Rev. Lett. 78, 5 (1997).

${ }^{18}$ G. Bimonte, Phys. Rev. Lett. 96, 160401 (2006).

${ }^{19}$ J. K. Gimzewski, Ch. Gerber, E. Meyer, and R. R. Schlittler, Chem. Phys. Lett. 217, 589 (1994).

${ }^{20}$ J. R. Barnes, R. J. Stephenson, C. N. Woodburn, S. J. O. Shea, M. E. Welland, T. Rayment, J. K. Gimzewski, and Ch. Gerber, Rev. Sci. Instrum. 65, 3793 (1994).

${ }^{21}$ J. Varesi et al., Appl. Phys. Lett. 71, 306 (1997).

${ }^{22}$ P. G. Datskos, N. V. Lavrik, and S. Rajic, Rev. Sci. Instrum. 75, 1134 (2004).

${ }^{23}$ U. Mohideen and A. Roy, Phys. Rev. Lett. 81, 4549 (1998).

${ }^{24}$ The conductance between a sphere and a flat substrate is approximately twice the conductance between two spheres of same diameter at the same value of gap. This is a result of the proximity approximation, which is frequently used in computing forces between curved surfaces based on the results of forces between parallel surfaces. Since we do not have a rigorous theory for near-field radiative transfer between a sphere and a substrate, this approximation is a necessary compromise.

${ }^{25}$ S. Shen, A. Narayanaswamy, S. Goh, and G. Chen, Appl. Phys. Lett. 92, 063509 (2008).

${ }^{26}$ A. Narayanaswamy, Ph.D. thesis, Massachusetts Institute of Technology, 2007.

${ }^{27}$ C. F. Bohren and D. R. Huffman, Absorption and Scattering of Light by Small Particles (Wiley, New York, 1983).

${ }^{28}$ R. Siegel and J. Howell, Thermal Radiation Heat Transfer (Taylor \& Francis, London, 2001).

${ }^{29}$ J. Blocki, J. Randrup, W. J. Swiatecki, and C. F. Tsang, Ann. Phys. (N.Y.) 105, 427 (1977).

${ }^{30}$ B. V. Derjaguin, I. I. Abrikosova, and E. M. Lifshitz, Q. Rev., Chem. Soc. 10, 295 (1956). 